\title{
Symbolic Algorithms for the Painlevé Test, Special Solutions, and Recursion Operators for Nonlinear PDEs.
}

\author{
D. Baldwin, W. Hereman, and J. Sayers
}

\begin{abstract}
This paper discusses the algorithms and implementations of three MATHEMATICA packages for the study of integrability and the computation of closed-form solutions of nonlinear polynomial PDEs.

The first package, PainleveTest,m, symbolically performs the Painlevé integrability test. The second package, PDESpecialsolutions.m, computes exact solutions expressible in hyperbolic or elliptic functions. The third package, PDERecursion0perator.m, generates and tests recursion operators.
\end{abstract}

\section{Introduction}

The investigation of complete integrability of nonlinear partial differential equations (PDEs) is a nontrivial matter [12]. Likewise, finding the explicit form of solitary wave and soliton solutions requires tedious, unwieldy computations which are best performed using computer algebra systems. For example, the symbolic conputation of solitons with Hirota's direct method and the homogenization method are covered in $[13, \mathbf{1 6}]$.

Recently, progress has been made using MATHEMATICA and MAPLE in applying the inverse scattering transform (IST) method to compute solitons for the CamassaHolm equation [17]. Before applying the IST method (a nontrivial exercise in analysis!), one would like to know if the PDE is completely integrable or what elementary travelling wave solutions exist. This is where the symbolic algorithms and packages presented in this paper come into play.

In this paper we introduce three algorithms and related MATHEMATICA packages [4] which may greatly aid the investigation of integrability and the search for exact solutions. In Section 2 we present the algorithm for the well-known Painlevé integrability test $[\mathbf{1}, \mathbf{5}, \mathbf{6}]$, which was recently implemented as PainleveTest.m. Section 3 outlines the algorithm behind PDESpecialsolutions . m, which allows one

2000 Mathematics Subject Classification. Primary 35Q53, 37K10; Secondary 35K40, $37 \mathrm{~K} 05$. This material is based upon work supported by the National Science Foundation (NSF) under Grants Nos. DMS-9732069. DMS-9912293 and CCR-9901929.

This is the final form of the paper. 
to automatically compute exact solutions expressible in hyperbolic or elliptic functions; full details are presented in [3]. In Section 4 we give an algorithm for computing and testing recursion operators [11]; the package PDERecursionOperator m alltomates the steps. The latter package builds on the code InvariantsSymetries.m [9], which computes conserved densities, finxes, and generalized symmetries for polynomial equations. Our codes are publicly available, see [4].

In Sections 2 and 3 we consider systems of $M$ polynonial differential equations,

$$
\Delta\left(\mathbf{u}(\mathbf{x}), \mathbf{u}^{\prime}(\mathbf{x}), \mathbf{u}^{\prime \prime}(\mathbf{x}), \ldots, \mathbf{u}^{(m)}(\mathbf{x})\right)=\mathbf{0}
$$

where the dependent variable $\mathbf{u}$ has $M$ components $u_{i}$, the independent variable $\mathbf{x}$ has $N$ components $x_{j}$, and $\mathbf{u}^{(m)}(\mathbf{x})$ denotes the collection of mixed derivative terms of order $m$. We assume that any arbitrary coefficients parameterizing the system are strictly positive and denoted by lower-case Greek letters. However, the algorithm in Section 4 only applies to a special case of (1.1), namely evolution equations in $(1+1)$ dimensions.

Two carefully selected examples will illustrate the algorithms. The first example is the Kaup-Kupershmidt (KK) equation (see e.g. $[\mathbf{8 , 2 2}]$ ),

$$
u_{t}=5 u^{2} u_{x}+\frac{25}{2} u_{x} u_{2 x}+5 u u_{3 x}+u_{5 x} .
$$

The second example is the Hirota-Satsuma system of coupled Korteweg-de Vries (KdV) equations (see e.g. [1]).

$$
\begin{gathered}
u_{t}=\beta\left(6 u u_{x}+u_{3 x}\right)-2 v v_{x}, \quad \beta>0 . \\
v_{t}=-3 u v_{x}-v_{3 x} .
\end{gathered}
$$

The computations for both examples will be done by the software.

\section{The Painlevé test}

The Painlevé test verifies whether a system of ODEs or PDEs satisfies the necessary conditions for having the Painleve property. There is some variation in what is meant by the Painlevé property [18]. As defined in [2], for a PDE to have the Painlevé property, all ODE reductions of the PDE must have the Painlevé property. While [2] requires that all movable singularities of all solutions are poles, the more general definition used by Painlevé himself requires that all solutions of the ODE are single-valued around all movable singularities. A later version [23] allows testing of PDEs directly without having to reduce them to ODEs. For a thorough discussion of the Painlevé property, see $[\mathbf{5}, \mathbf{6}]$.

Definition 2.1. A PDE has the Painleve property if its solutions in the complex plane are single-valued in the neighborhood of all its movable singularities.

2.1. Algorithm and implementation. Following [23], we assume a Laurent expansion for the solution

$$
u_{2}(\mathbf{x})=g^{\alpha_{2}}(\mathbf{x}) \sum_{k=0}^{\infty} u_{i, k}(\mathbf{x}) g^{k}(\mathbf{x}), \quad u_{i, 0}(\mathbf{x}) \neq 0 \quad \text { and } \quad \alpha_{i} \in \mathbb{Z} .
$$

where at least one of the leading exponents $\alpha_{i}$ is a negative integer and $u_{i, k}(\mathbf{x})$ is an analytic function in the neighborhood of $g(\mathbf{x})$. The solution should be single-valned in the neighborhood of the non-characteristic, movable singular manifold $g(\mathbf{x})=0$, which can be viewed as the surface of the movable poles in the complex plane. 
The algorithm for the Painleve test is composed of the following three steps:

Step 1 (Determine the dominant behavior). It suffices to substitute

$$
u_{i}(\mathbf{x})=u_{i, 0}(\mathbf{x}) g^{\alpha_{i}}(\mathbf{x})
$$

into (1.1) to determine the leading exponents $\alpha_{i}$ (one of which must be a negative integer) and the function $u_{i, 0}(\mathbf{x})$. In the resulting polynomial system, equating every two possible lowest exponents of $g(\mathbf{x})$ in each equation gives a linear system to determine $\alpha_{i}$. The linear system is then solved for $\alpha_{i}$.

If one or more exponents $\alpha_{i}$ remain undetermined, we assign integer values to the free $\alpha_{i}$ (if applicable, up to a user-specified non-negative boundary, DominantBehaviorMax, with default value -1) so that every equation in (1.1) has at least two different terms with equal lowest exponents. Once $\alpha_{i}$ is known, we substitute (2.2) back into (1.1) and solve for $u_{i, 0}(\mathbf{x})$.

Step 2 (Determine the resonances). For each $\alpha$, and $u_{i, 0}(\mathbf{x})$, we calculate the integers $r$ for which $u_{t, r}(\mathbf{x})$ is an arbitrary function in (2.1). We substitute

$$
u_{i}(\mathbf{x})=u_{i, 0}(\mathbf{x}) g^{\alpha_{i}}(\mathbf{x})+u_{i, r}(\mathbf{x}) g^{\alpha_{i}+r}(\mathbf{x})
$$

into (1.1). keeping only the lowest order terms in $g(\mathbf{x})$, and requiring that the coefficients of $u_{i, r}(\mathbf{x})$ equate to zero. This is dorle by computing the roots for $r$ of $\operatorname{det} Q=0$, where the $M \times M$ matrix $Q$ satisfies

$$
Q \cdot \mathbf{u}_{r}=0, \quad \mathbf{u}_{r}=\left(\begin{array}{llll}
u_{1, r} & u_{2, r} & \cdots & u_{M, r}
\end{array}\right)^{\mathrm{T}} .
$$

Step 3 (Find the constants of integration and check compatibility conditions). For the system to possess the Painlevé property, the arbitrariness of $u_{i, r}(\mathbf{x})$ must be verified up to the highest resonance level. That is, all compatibility conditions must be trivially satisfied. This is done by substituting

$$
u_{i}(\mathbf{x})=g^{\alpha_{x}}(\mathbf{x}) \sum_{k=0}^{r_{\text {Max }}} u_{i, k}(\mathbf{x}) g^{k}(\mathbf{x})
$$

into (1.1), where $r_{\mathrm{Max}}$ is the highest positive integer resonance. For the system to have the Painleve property, there must be as many arbitrary constants of integration at resonance levels as resonances at that level. Furthermore, all constants of integration $u_{i, k}(\mathbf{x})$ at non-resonance levels must be unambiguously determined.

\subsection{Examples of the Painlevé test.}

Example 2.1 (Kaup-Kupershmidt). To determine the dominant behavior, we substitute (2.2) into (1.2) and pull off the exponents of $g(\mathbf{x})$ (see Table 1).

Removing duplicates and non-dominant exponents, we are left with

$$
\left\{\alpha_{1}-5,2 \alpha_{1}-3,3 \alpha_{1}-1\right\} \text {. }
$$

Considering all possible balances of two or more exponents leads to $\alpha_{1}=-2$.

Substituting $u(\mathbf{x})=u_{1,0}(\mathbf{x}) g^{-2}(\mathbf{x})$ into $(1.2)$ and solving for $u_{1,0}(\mathbf{x})$ gives $u$

$$
u_{1,0}(\mathbf{x})=-24 g_{x}^{2}(\mathbf{x}) \text { and } u_{1,0}(\mathbf{x})=-3 g_{x}^{2}(\mathbf{x}) .
$$

For the first branch, substituting $u(\mathbf{x})=-24 g_{x}^{2}(\mathbf{x}) g^{-2}(\mathbf{x})+u_{1, r}(\mathbf{x}) g^{r-2}(\mathbf{x})$ into (1.2), keeping the lowest order terms, and taking the coefficient of $u_{1, r}(\mathbf{x})$, gives

$$
-2(r+7)(r+1)(r-6)(r-10)(r-12) g_{r}^{5}(\mathbf{x})=0 .
$$


TABLE 1. The exponents of $g(\mathbf{x})$ for (1.2).

\begin{tabular}{c|c} 
Term & Exponents of $g(\mathbf{x})$ with duplicates removed \\
\hline \hline$u_{t}$ & $\alpha_{1}-1$ \\
\hline$u_{5 x}$ & $\alpha_{1}-5, \alpha_{1}-4, \alpha_{1}-3, \alpha_{1}-2, \alpha_{1}-1$ \\
\hline $5 u u_{3 x}$ & $2 \alpha_{1}-3,2 \alpha_{1}-2,2 \alpha_{1}-1$ \\
\hline$\frac{25}{2} u_{x} u_{2 x}$ & $2 \alpha_{1}-3,2 \alpha_{1}-2$ \\
\hline $5 u^{2} u_{x}$ & $3 \alpha_{1}-1$
\end{tabular}

Hence, $r=-7,-1,6,10,12$. The first branch is not a principal branch due to the presence of a negative resonance other than $r=-1$. The latter is called the universal resonance and corresponds to the arbitrariness of the manifold function $g(\mathbf{x})$. In $[\boldsymbol{7}]$, a perturbative Painlevé approach is presented which properly treats negative integer resonances. As is common practice, our algorithm ignores them.

The constants of integration at level $j$ are found by substituting (2.5) into (1.2), where $r_{\operatorname{Max}}=12$, and pulling off the coefficients of $g^{3}(\mathbf{x})$. The first few are

$$
\begin{aligned}
& \text { at } j=1: u_{1,1}(\mathbf{x})=24 g_{2 x}(\mathbf{x}), \\
& \text { at } j=2: u_{1,2}(\mathbf{x})=\frac{6 g_{2 x}^{2}(\mathbf{x})-8 g_{x}(\mathbf{x}) g_{3 x}(\mathbf{x})}{g_{x}^{2}(\mathbf{x})}, \\
& \text { at } j=3: u_{1,3}(\mathbf{x})=\frac{6 g_{2 x}^{3}(\mathbf{x})-8 g_{x}(\mathbf{x}) g_{2 x}(\mathbf{x}) g_{3 x}(\mathbf{x})+2 g_{x}^{2}(\mathbf{x}) g_{4 x}(\mathbf{x})}{g_{x}^{4}(\mathbf{x})} .
\end{aligned}
$$

The compatibility conditions are satisfied at resonance levels 6,10 , and 12 . The remaining constants of integration $u_{I, j}(\mathbf{x})$ were computed but not shown here.

Likewise, in thesecond branch, substitute $u(\mathbf{x})=-3 g_{x}^{2}(\mathbf{x}) g^{-2}(\mathbf{x})+u_{1, r}(\mathbf{x}) g^{r-2}(\mathbf{x})$ into (1.2). and proceed as before to get $r=-1,3,5.6,7$. Thus, the second branch is a principal branch. The constants of integration at $j=1,2$ and 4 are again found by substituting (2.5) into (1.2) and pulling of the coefficients of $g^{j}(\mathbf{x})$. This gives,

$$
\begin{aligned}
& \text { at } j=1: u_{1,1}(\mathbf{x})=3 g_{2 x}(\mathbf{x}), \\
& \text { at } j=2: u_{1,2}(\mathbf{x})=\frac{3 g_{2 x}^{2}(\mathbf{x})-4 g_{x}(\mathbf{x}) g_{3 x}(\mathbf{x})}{4 g_{x}^{2}(\mathbf{x})} \text {. }
\end{aligned}
$$

Coefficient $u_{1,4}$ at level $j=4$ is not shown here due to length. At the resonance levels $r=3,5,6,7$, the compatibility conditions happen to be satisfied. So, (1.2) passes the Painlevé test. It is well-known (see e.g. $[\mathbf{8 , 2 2}]$ ) that $(1.2)$ is completely integrable.

Example 2.2 (Hirota-Satsuma). The Hirota-Satsuma system illustrates the subtleties of determining the dominant behavior.

As in Example 2.1, we substitute (2.2) into (1.3) and pull off the exponents of $g(\mathbf{x})$ (listed in Table 2).

Removing non-dominant exponents and duplicates by term, we get $\left\{\alpha_{1}-\right.$ $\left.3,2 \alpha_{1}-1,2 \alpha_{2}-1\right\}$ from $\Delta_{1}$ and $\left\{\alpha_{2}-3, \alpha_{1}+\alpha_{2}-1\right\}$ from $\Delta_{2}$.

Equating the possible dominant exponents from $\Delta_{2}$ gives us $\alpha_{2}-3=\alpha_{1}+\alpha_{2}-1$ or $\alpha_{1}=-2$. Unexpectedly, $\alpha_{1}=-2$ balances two of the possible dominant terms 
TABle 2. The exponents of $g(\mathbf{x})$ for $(1.3)$.

\begin{tabular}{c|c} 
Term & Exponents of $g(\mathbf{x})$ \\
\hline \hline$u_{f}$ & $\alpha_{1}-1$ \\
\hline$-\beta u_{3 x}$ & $\alpha_{1}-3, \alpha_{1}-3, \alpha_{1}-3, \alpha_{1}-2, \alpha_{1}-2, \alpha_{1}-1$ \\
\hline$-6 \beta u u_{x}$ & $2 \alpha_{1}-1$ \\
\hline $2 v v_{x}$ & $2 \alpha_{2}-1$ \\
\hline \hline$v_{t}$ & $\alpha_{2}-1$ \\
\hline$v_{3 x}$ & $\alpha_{2}-3, \alpha_{2}-3, \alpha_{2}-3, \alpha_{2}-2, \alpha_{2}-2, \alpha_{2}-1$ \\
\hline $3 u v_{x}$ & $\alpha_{1}+\alpha_{2}-1$
\end{tabular}

in $\Delta_{1}$, and we are free to choose $\alpha_{2}$ such that

$$
2 \alpha_{1}-1 \leq 2 \alpha_{2}-1 \text { or }-2 \leq \alpha_{2} \text {. }
$$

Using the default value -1 for DominantBehaviorMax, we have $-2 \leq \alpha_{2} \leq-1$. Hence, $\alpha_{2}=-1$ or $\alpha_{2}=-2$.

Using the two solutions for $\alpha_{i}$ and solving for $u_{i, 0}$ results in

$$
\begin{aligned}
& \begin{cases}\alpha_{1}=-2, & u_{1,0}(\mathbf{x})=-4 g_{x}^{2}(\mathbf{x}), \\
\alpha_{2}=-2, & u_{2,0}(\mathbf{x})= \pm 2 \sqrt{6 \beta} g_{x}^{2}(\mathbf{x}),\end{cases} \\
& \begin{cases}\alpha_{1}=-2, & u_{1.0}(\mathbf{x})=-2 g_{x}^{2}(\mathbf{x}), \\
\alpha_{2}=-1, & u_{2,0}(\mathbf{x}) \text { arbitrary. }\end{cases}
\end{aligned}
$$

We substitute (2.3) into (1.3) while using the results for $\alpha_{i}$ and $u_{i, 0}(\mathbf{x})$. For (2.15), substituting $u(\mathbf{x})=-4 g_{x}^{2}(\mathbf{x}) g^{-2}(\mathbf{x})+u_{1, r}(\mathbf{x}) g^{r-2}(\mathbf{x})$ and $v(\mathbf{x})= \pm 2 \sqrt{6} \beta g_{x}^{2}(\mathbf{x}) g^{-2}(\mathbf{x})$ $+u_{2, r}(\mathbf{x}) g^{r-2}(\mathbf{x})$ into (1.3) and keeping the most singular terms gives

$$
Q \cdot \mathbf{u}_{r}=\left(\begin{array}{cc}
-(r-4)\left(r^{2}-5 r-18\right) \beta g_{x}^{3}(\mathbf{x}) & \pm 12 \sqrt{6 \beta} g_{x}^{3}(\mathbf{x}) \\
\mp 4(r-4) \sqrt{6 \beta} g_{x}^{3}(\mathbf{x}) & (r-2)(r-7) r g_{x}^{3}(\mathbf{x})
\end{array}\right)\left(\begin{array}{l}
u_{1, r}(\mathbf{x}) \\
u_{2, r}(\mathbf{x})
\end{array}\right)=0 .
$$

Setting

$$
\operatorname{det} Q=-\beta(r+2)(r+1)(\tau-3)(r-4)(r-6)(r-8) g_{x}^{6}(\mathbf{x})=0
$$

yields $r=-2,-1,3,4,6,8$. This is not a principal branch.

As in the previous example, the constants of integration at level $j$ are found by substituting (2.5) into (1.3) and pulling off the coefficients of $g^{j}(\mathbf{x})$. At $j=1$,

$$
\left(\begin{array}{cc}
-66 \beta g_{x}^{3}(\mathbf{x}) & \mp 12 \sqrt{6 \beta} g_{x}^{3}(\mathbf{x}) \\
\mp 12 \sqrt{6 \beta} g_{x}^{3}(\mathbf{x}) & 6 g_{x}^{3}(\mathbf{x})
\end{array}\right)\left(\begin{array}{l}
u_{1,1}(\mathbf{x}) \\
u_{2,1}(\mathbf{x})
\end{array}\right)=\left(\begin{array}{c}
-120 \beta g_{x}^{3}(\mathbf{x}) g_{2 x}(\mathbf{x}) \\
\mp 60 \sqrt{6 \beta} g_{x}^{3}(\mathbf{x}) g_{2 x}(\mathbf{x})
\end{array}\right) .
$$

Thus,

$$
u_{1, I}(\mathbf{x})=4 g_{2 x}(\mathbf{x}), \quad u_{2,1}(\mathbf{x})= \pm 2 \sqrt{6} \beta g_{2 x}(\mathbf{x}) .
$$


At $j=2$,

$$
\begin{aligned}
& u_{1,2}(\mathbf{x})=\frac{3 g_{2 x}^{2}(\mathbf{x})-g_{x}(\mathbf{x})\left(g_{t}(\mathbf{x})+4 g_{3 x}(\mathbf{x})\right)}{3 g_{x}^{2}(\mathbf{x})} \\
& u_{2,2}(\mathbf{x})= \pm \frac{3 \beta g_{2 x}^{2}(\mathbf{x})-4 \beta g_{x}(\mathbf{x}) g_{3 x}(\mathbf{x})-(1+2 \beta) g_{t}(\mathbf{x}) g_{x}(\mathbf{x})}{\sqrt{6 \beta} g_{x}^{2}(\mathbf{x})}
\end{aligned}
$$

The remaining constants of integration are omitted due to length. The compatibility conditions at $r=3$ and 4 are satisfied. At $r=6$ and $r=8$, the compatibility conditions require $\beta=\frac{1}{2}$.

Likewise, for $(2.16)$. the resonances following from the substitution of $u(\mathbf{x})=$ $-2 g_{x}^{2}(\mathbf{x}) g^{-2}(\mathbf{x})+u_{1, r}(\mathbf{x}) g^{r-2}(\mathbf{x})$ and $v(\mathbf{x})=u_{2,0}(\mathbf{x}) g^{-2}(\mathbf{x})+u_{2, r}(\mathbf{x}) g^{r-1}(\mathbf{x})$ into (1.3) are $r=-1,0,1,4,5.6$. This is a principal branch. The zero resonance explains the arbitrariness of $u_{2,0}(\mathbf{x})$. Similarly, we computed all constants of integration, but ran into a compatibility conditions at $r=5$ and $r=6$. which requires $\beta=\frac{1}{2}$. Therefore, (1.3) passes the Painlevé test if $\beta=\frac{1}{2}$, a fact confirmed by other analyses of integrability [1].

\section{Travelling wave solutions in hyperbolic or elliptic functions}

The traveling wave solutions of many nonlinear ODEs and PDEs from soliton theory (and elsewhere) can be expressed as polynomials of hyperbolic or elliptic functions. For instance, the bell shaped sech-solutions and kink shaped tanhsolutions model wave phenomena in fuid dynamics, plasmas, elastic media, electrical circuits, optical fibers, chemical reactions, bio-genetics, etc. A discussion is given in [15], while a multitude of references to tanh-based techniques and applications can be found in $[3,19]$.

In this section we discuss the tanh-, sech-, cn- and sn-methods as they apply to nonlinear polynomial systems of ODEs and PDEs in multi-dimensions.

3.1. Algorithm and implementation. All four flavors of the algorithm share the same five basic steps.

Step 1 (Transform the PDE into a nonlinear ODE). We seek solutions in the traveling frame of reference,

$$
\xi=\sum_{j=1}^{N} c_{j} x_{j}+\delta_{3}
$$

where the components $c_{3}$ of the wave vector and the phase $\delta$ are constants.

In the tanh inethod, we seek polynomial solutions expressible in hyperbolic tangent, $T=\tanh \xi$. Based on the identity $\cosh ^{2} \xi-\sinh ^{2} \xi=1$,

$$
\begin{aligned}
\tanh ^{\prime} \xi & =\operatorname{sech}^{2} \xi=1-\tanh ^{2} \xi, \\
\tanh ^{\prime \prime} \xi & =-2 \tanh \xi+2 \tanh ^{3} \xi, \text { etc. }
\end{aligned}
$$

Therefore, the first and all higher-order derivatives of $T=\tanh \xi$ are polynomial in $T$. Consequently, repeatedly applying the chain rule,

$$
T=\tanh (\xi): \frac{\partial \bullet}{\partial x_{j}}=\frac{\partial \xi}{\partial x_{j}} \frac{d T}{d \xi} \frac{d \bullet}{d T}=c_{j}\left(1-T^{2}\right) \frac{d \bullet}{d T}
$$

transforms (1.1) into a coupled system of nonlinear ODEs. 
TABLE 3. Values for $R(F)$ in $(3.11)$.

\begin{tabular}{c|c}
$F$ & $R(F)$ \\
\hline \hline$T$ & 0 \\
\hline$S$ & $1-S^{2}$ \\
\hline $\mathrm{CN}$ & $\left(1-\mathrm{CN}^{2}\right)\left(1-m+m \mathrm{CN}^{2}\right)$ \\
\hline $\mathrm{SN}$ & $\left(1-\mathrm{SN}^{2}\right)\left(1-m \mathrm{SN}^{2}\right)$
\end{tabular}

Similarly, using the identity $\tanh ^{2} \xi+\operatorname{sech}^{2} \xi=1$, we get

$$
\operatorname{sech}^{\prime} \xi=-\operatorname{sech} \xi \tanh \xi=-\operatorname{sech} \xi \sqrt{1-\operatorname{sech}^{2} \xi} .
$$

Likewise, for Jacobi's elliptic functions with modulus $m$, we use the identities

$$
\operatorname{sn}^{2}(\xi ; m)=1-\mathrm{cn}^{2}(\xi ; m), \text { and } \operatorname{dn}^{2}(\xi ; m)=1-m+m \mathrm{cn}^{2}(\xi ; m) .
$$

to write, for example, $\mathrm{cn}^{\prime}$ in terms of $\mathrm{cn}$ :

$$
\mathrm{cn}^{\prime}(\xi ; m)=-\mathrm{sn}(\xi ; m) \mathrm{dn}(\xi ; m)=-\sqrt{\left(1-\mathrm{cn}^{2}(\xi ; m)\right)\left(1-m+m \mathrm{cn}^{2}(\xi ; m)\right)}
$$

Then, repeatedly applying the chain rules,

$$
\begin{aligned}
S & =\operatorname{sech}(\xi): \frac{\partial \bullet}{\partial x_{j}}=-c_{j} S \sqrt{1-S^{2}} \frac{d \bullet}{d S} . \\
\mathrm{CN} & =\operatorname{cn}(\xi ; m): \frac{\partial \bullet}{\partial x_{j}}=-c_{j} \sqrt{\left(1-\mathrm{CN}^{2}\right)\left(1-m+m \mathrm{CN}^{2}\right)} \frac{d \bullet}{d \mathrm{CN}}, \\
\mathrm{SN} & =\operatorname{sn}(\xi ; m): \frac{\partial \bullet}{\partial x_{j}}=c_{j} \sqrt{\left(1-\mathrm{SN}^{2}\right)\left(1-m \mathrm{SN}^{2}\right)} \frac{d \bullet}{d S N} .
\end{aligned}
$$

we transform (1.1) into a coupled system of nonlinear ODEs of the form

$$
\mathbf{\Gamma}\left(F, \mathbf{u}(F), \mathbf{u}^{\prime}(F) \ldots\right)+\sqrt{R(F)} \boldsymbol{\Pi}\left(F, \mathbf{u}(F), \mathbf{u}^{\prime}(F), \ldots\right)=\mathbf{0} .
$$

where $F$ is either $T, S, C N$, or SN, and $R(F)$ is defined in Table 3 .

Step 2 (Determine the degree of the polynomial solutious). Since we seek polynomial solutions

$$
U_{i}(F)=\sum_{j=0}^{M_{i}} a_{2 j} F^{j},
$$

the leading exponents $M_{i}$ must be determined before the $a_{i}$ can be computed. The process for determining $M_{i}$ is quite similar to the one for finding $\alpha_{i}$ in Section 2.

Substituting $U_{i}(F)$ into (3.11), the coefficients of every power of $F$ in every equation must vanish. In particular, the highest degree terms must vanish. Since the highest degree terms only depend on $F^{M_{i}}$ in (3.12), it suffices to substitute $U_{i}(F)=F^{M_{+}}$into (3.11). Doing so. we get

$$
\mathbf{P}(F)+\sqrt{R(F)} \mathbf{Q}(F)=0,
$$




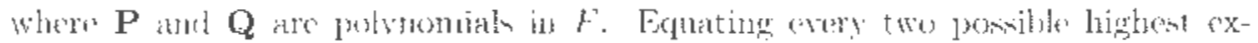

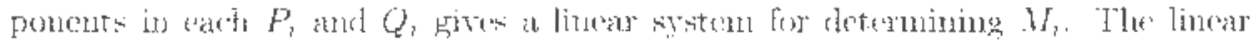

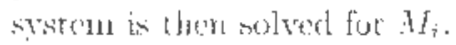

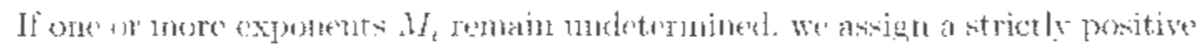

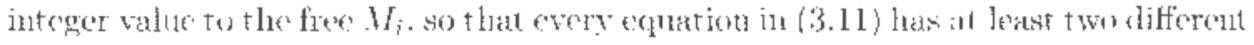

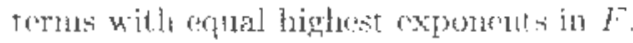

Step 3 (Derive the algebraic system for the coefficients $u_{1,1}$ ). Tu gerert-

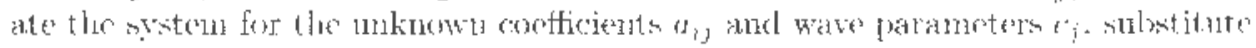

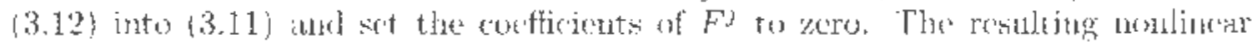

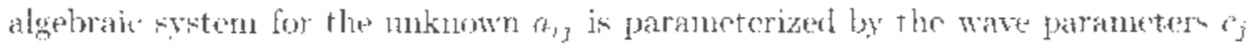

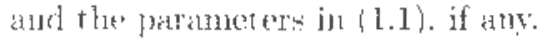

Step 4 (Solve the nonlinear paraneterized algebraic systern). Thr

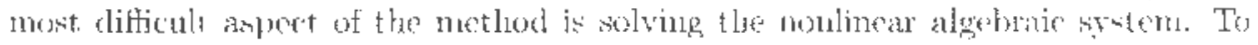

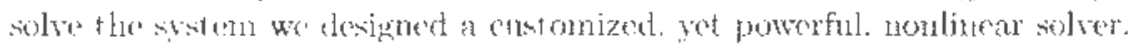

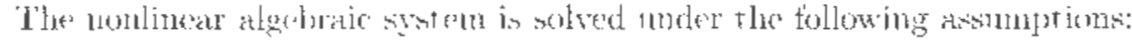

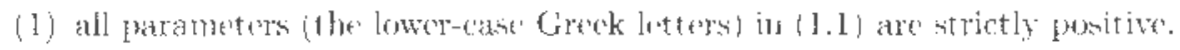

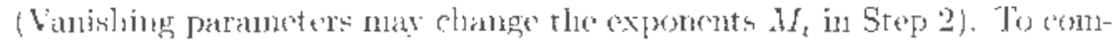

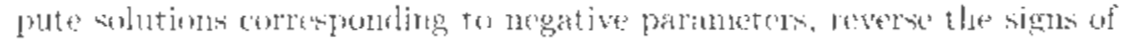
the parametedts in (1.1).

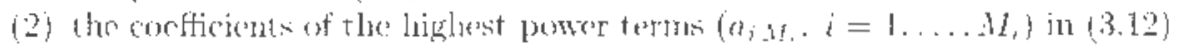
are all romero ! for consistone with srep 2 !

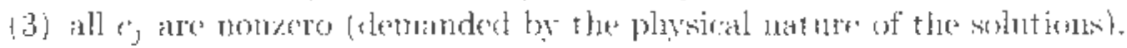

Step 5 (Build and test solutions). Sulstilu the sulutions from Ste tinto

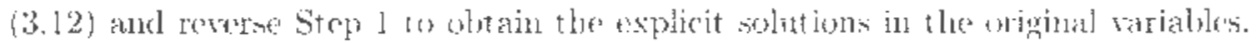
It is prodera to test the solutions by substitimg theme into (1.1). which we do.

\subsection{Examples of travelling wave solutions.}

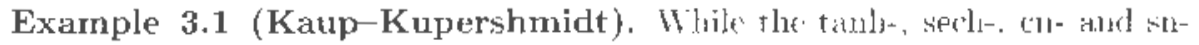

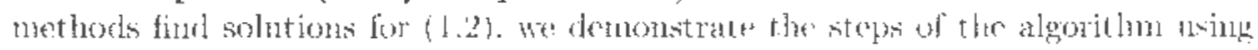

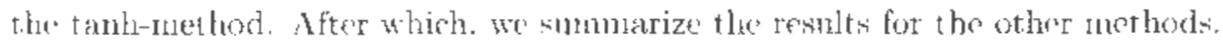

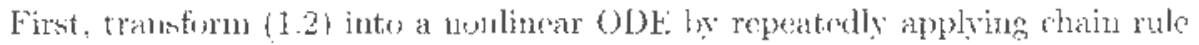
(3.4). The renlting ODF is

$(3,14) 20 T^{41}$

$$
\begin{aligned}
& +r \cdot\left[10 C^{2} Q^{\prime \prime}+r^{2}\left[2 \pi\left(T^{2}-1\right) U^{\prime \prime}\left[2 T C^{\prime \prime}+1 T^{2}-1\right) U^{\prime \prime}\right.\right.
\end{aligned}
$$

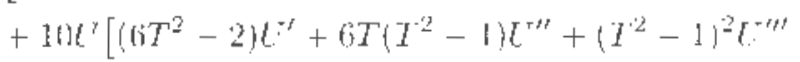

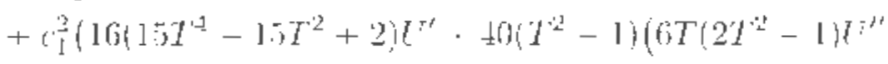

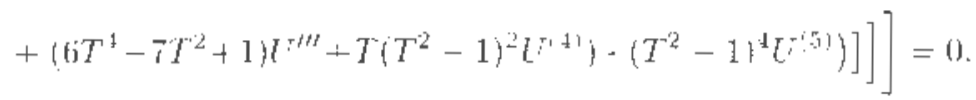

whele $T=$ tanlel $s)$ and $T^{r}=(C(T)$.

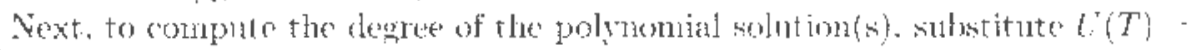

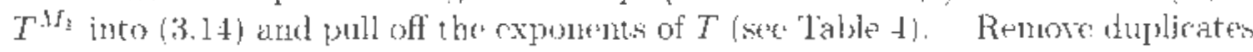
and ron-dominatit oxponents. to get

$$
\left\{3 M_{1}-1.2 H_{1}+1 . M_{1}+3\right\} .
$$


TABLE 4. The exponents of $T$ after substituting $U(T)=T^{M_{1}}$.

\begin{tabular}{c|c} 
Term & Exponents of $T$ with duplicates removed \\
\hline$u_{t}$ & $M_{1}-1$ \\
\hline$u^{2} u_{x}$ & $3 M_{1}-1$ \\
\hline$\frac{25}{2} u_{x} u_{2 x}$ & $2 M_{1}+1,2 M_{1}-1,2 M_{1}-3$ \\
\hline $5 u u_{3 x}$ & $2 M_{1}+1,2 M_{1}-1,2 M_{1}-3$ \\
\hline$u_{5 x}$ & $M_{1}+3, M_{1}+1, M_{1}-1, M_{1}-3, M_{1}-5$
\end{tabular}

Consider all possible balances of two or more exponents to find $M_{1}=2$.

Substitute

$$
U(T)=a_{10}+a_{11} T+a_{12} T^{2}
$$

into (3.14) and equate the coefficients of $T^{j}$ to zero (where $i=0,1, \ldots, 5$ ) to get

$$
\begin{gathered}
\left(a_{12}+3 c_{1}^{2}\right)\left(a_{12}+24 c_{1}^{2}\right)=0, \\
a_{11}\left(5 a_{12}^{2}+55 a_{12} c_{1}^{2}+24 c_{1}^{4}\right)=0, \\
a_{11}\left(5 a_{10}^{2} c_{1}-10 a_{10} c_{1}^{3}+25 a_{12} c_{1}^{3}+16 c_{1}^{5}+c_{2}\right)=0, \\
a_{11}\left(a_{11}^{2}+6 a_{10} a_{12}+6 a_{10} c_{1}^{2}-48 a_{12} c_{1}^{2}-24 c_{1}^{4}\right)=0, \\
4 a_{11}^{2} a_{12}+4 a_{10} a_{12}^{2}+11 a_{11}^{2} c_{1}^{2}+24 a_{10} a_{12} c_{1}^{2}-56 a_{12}^{2} c_{1}^{2}-192 a_{12} c_{1}^{4}=0, \\
10 a_{10} a_{11}^{2} c_{1}+10 a_{10}^{2} a_{12} c_{1}-35 a_{11}^{2} c_{1}^{3} \\
-80 a_{10} a_{12} c_{1}^{3}+50 a_{12}^{2} c_{1}^{3}+272 a_{12} c_{1}^{5}+2 a_{12} c_{2}=0 .
\end{gathered}
$$

Solve the nonlinear algebraic system with the assumption that $a_{12}, c_{1}$, and $c_{2}$ are all nonzero. Two solutions are obtained:

$$
\left\{\begin{array} { l l } 
{ a _ { 1 0 } = 1 6 c _ { 1 } ^ { 2 } , } & { a _ { 1 1 } = 0 , } \\
{ a _ { 1 2 } = - 2 4 c _ { 1 } ^ { 2 } , } & { c _ { 2 } = - 1 7 6 c _ { 1 } ^ { 5 } , }
\end{array} \text { and } \left\{\begin{array}{ll}
a_{10}=2 c_{1}^{2}, & a_{11}=0, \\
a_{12}=-3 c_{1}^{2}, & c_{2}=-c_{1}^{5} .
\end{array}\right.\right.
$$

where $c_{1}$ is arbitrary.

Substitute the solutions into $(3.16)$ and return to $u(x, t)$ to get

$$
\begin{aligned}
& u(x, t)=16 c_{1}^{2}-24 c_{1}^{2} \tanh ^{2}\left(c_{1} x-176 c_{1}^{5} t+\delta\right) . \\
& u(x, t)=2 c_{1}^{2}-3 c_{1}^{2} \tanh ^{2}\left(c_{1} x-c_{1}^{5} t+\delta\right) .
\end{aligned}
$$

Using the sech-method, one finds

$$
\begin{aligned}
& u(x, t)=-8 c_{1}^{2}+24 c_{1}^{2} \operatorname{sech}^{2}\left(c_{1} x-176 c_{1}^{5} t+\delta\right), \\
& u(x, t)=-c_{1}^{2}+3 c_{1}^{2} \operatorname{sech}^{2}\left(c_{1} x-c_{1}^{5} t+\delta\right) .
\end{aligned}
$$

Alternatively, the latter solutions can be found directly from the tanh-solutions by using the identity $\tanh ^{2} \xi=1-\operatorname{sech}^{2} \xi$.

Using the sn-method, one gets

$$
\begin{aligned}
& u(x, t)=8 c_{1}^{2}\left[1+m-3 m \operatorname{sn}^{2}\left(c_{1} x-176 c_{1}^{5}\left(m^{2}-m+1\right) t+\delta ; m\right)\right], \\
& u(x, t)=c_{1}^{2}\left[1+m-3 m \sin ^{2}\left(c_{1} x-c_{1}^{5}\left(m^{2}-m+1\right) t+\delta ; m\right)\right] .
\end{aligned}
$$


The cn-solutions computed with the cn-method are not explicitly shown since they can be obtained from the sn-solutions using the identity $\operatorname{sn}^{2} \xi=1-\operatorname{cn}^{2} \xi$.

Example 3.2 (Hirota-Satsuma). As in the previous example. the tanh-, sech-, cn- and sn-methods all find solutions for (1.3). In this example, however, we will illustrate the steps using the sech-method.

Transform (1.3) into a coupled system of ODEs, apply the chain rule (3.8) and cancel the common $S \sqrt{1-S^{2}}$ factors to get

$$
\begin{aligned}
& c_{2} U_{1}^{\prime}-6 \beta c_{1} U_{1} U_{1}^{\prime}- \beta c_{1}^{3}\left\{\left(1-6 S^{2}\right) U_{1}^{\prime}\right. \\
&\left.+3 S\left(1-2 S^{2}\right) U_{1}^{\prime \prime}+S^{2}\left(1-S^{2}\right) U_{1}^{\prime \prime \prime}\right]+2 c_{1} U_{2} U_{2}^{\prime}=0 . \\
& c_{2} U_{2}^{\prime}+3 c_{1} U_{1} U_{2}^{\prime}+c_{1}^{3}\left[\left(1-6 S^{2}\right) U_{2}^{\prime}+3 S\left(1-2 S^{2}\right) U_{2}^{\prime \prime}+S^{2}\left(1-S^{2}\right) U_{2}^{\prime \prime \prime}\right]=0 .
\end{aligned}
$$

To find the degree of the polynomials, substitute $U_{1}(S)=S^{M_{4}} \cdot U_{2}(S)=S^{M M_{2}}$ into (3.25) and equate the highest exponents from $\Delta_{2}$ to get

$$
M_{1}+M_{2}-1=1+M_{2} \text { or } M_{1}=2 \text {. }
$$

The maximal exponents coming from $\Delta_{1}$ are $2 M_{1}-1$ (from the $U_{1} U_{1}^{\prime}$ term), $M_{1}+1$ (from $U_{1}^{\prime \prime \prime}$ ), and $2 M_{2}-1$ (from $U_{2} U_{2}^{\prime}$ ).

Since $M_{1}=2$ balances at least two of the possible dominant exponents in $\Delta_{1}$, namely $2 M_{1}-1$ and $M_{1}+1$, one is again left with $1 \leq M_{2} \leq M_{1}=2$, or

$$
\begin{gathered}
\begin{cases}M_{1}=2, & U_{1}(S)=a_{10}+a_{11} S+a_{12} S^{2}, \\
M_{2}=1, & U_{2}(S)=a_{20}+a_{21} S,\end{cases} \\
\begin{cases}M_{1}=2, & U_{1}(S)=a_{10}+a_{11} S+a_{12} S^{2} . \\
M_{2}=2, & U_{2}(S)=a_{20}+a_{21} S+a_{22} S^{2} .\end{cases}
\end{gathered}
$$

To derive the algebraic system for $a_{i j}$, substitute (3.27) into (3.25), cancel common numerical factors, and organize the equations (according to complexity):

$$
\begin{gathered}
a_{11} a_{21} c_{1}=0, \\
\beta a_{11} c_{1}\left(3 a_{12}-c_{1}^{2}\right)=0, \\
3 a_{12} c_{1}\left(a_{12}-2 c_{1}^{2}\right)=0, \\
a_{21} c_{1}\left(a_{12}-2 c_{1}^{2}\right)=0, \\
a_{21}\left(3 a_{10} c_{1}+c_{1}^{3}+c_{2}\right)=0, \\
6 \beta a_{10} a_{11} c_{1}-2 a_{20} a_{21} c_{1}+\beta a_{11} c_{1}^{3}-a_{12} c_{2}=0, \\
3 \beta a_{11}^{2} c_{1}+6 \beta a_{10} a_{12} c_{1}-a_{21}^{2} c_{1}+4 \beta a_{12} c_{1}^{3}-a_{12} c_{2}=0 .
\end{gathered}
$$


Sinilarly, after substitution of (3.28) into (3.25), one gets

$$
\begin{gathered}
a_{22} c_{1}\left(a_{12}-4 c_{1}^{2}\right)=0, \\
a_{21}\left(3 a_{10} c_{1}+c_{1}^{3}+c_{2}\right)=0, \\
c_{1}\left(a_{12} a_{21}+2 a_{11} a_{22}-2 a_{21} c_{1}^{2}\right)=0 . \\
c_{1}\left(3 \beta a_{11} a_{12}-a_{21} a_{22}-\beta a_{11} c_{1}^{2}\right)=0, \\
c_{1}\left(3 \beta a_{12}^{2}-a_{22}^{2}-6 \beta a_{12} c_{1}^{2}\right)=0, \\
6 \beta a_{10} a_{11} c_{1}-2 a_{20} a_{21} c_{1}+\beta a_{11} c_{1}^{3}-a_{11} c_{2}=0, \\
3 a_{11} a_{21} c_{1}+6 a_{10} a_{22} c_{1}+8 a_{22} c_{1}^{3}+2 a_{22} c_{2}=0 . \\
3 \beta a_{11}^{2} c_{1}+6 \beta a_{10}^{2} a_{12} c_{1}-a_{21}^{2} c_{1}-2 a_{20} a_{22} c_{1}+4 \beta a_{12} c_{1}^{3}-a_{12} c_{2}=0 .
\end{gathered}
$$

Since $a_{12}, a_{21}, \beta, c_{1}$, and $c_{2}$, are nonzero, the solution of (3.29) is

$$
\begin{cases}a_{10}=-\left(c_{1}^{3}+c_{2}\right) /\left(3 c_{1}\right), & a_{20}=0, \\ a_{11}=0, & a_{21}= \pm \sqrt{4 \beta c_{1}^{4}-2(1+2 \beta) c_{1} c_{2},} \\ a_{12}=2 c_{1}^{2} . & \end{cases}
$$

For $a_{12}, a_{22}, \beta, c_{1}$, and $c_{2}$ nonzero, the solution of $(3.30)$ is

$$
\begin{cases}a_{10}=-\left(4 c_{1}^{3}+c_{2}\right) /\left(3 c_{1}\right), & a_{20}= \pm\left[4 \beta c_{1}^{3}+(1+2 \beta) c_{2}\right] /\left(c_{1} \sqrt{6 \beta}\right), \\ a_{11}=0, & a_{21}=0, \\ a_{12}=4 c_{1}^{2}, & a_{22}=\mp 2 c_{1}^{2} \sqrt{6 \beta} .\end{cases}
$$

The solutions of (1.3) involving sech are then

$$
\begin{aligned}
& u(x, t)=-\frac{c_{1}^{3}+c_{2}}{3 c_{1}}+2 c_{1}^{2} \operatorname{sech}^{2}\left(c_{1} x+c_{2} t+\delta\right), \\
& v(x, t)= \pm \sqrt{4 \beta c_{1}^{4}-2(1+2 \beta) c_{1} c_{2}} \operatorname{sech}\left(c_{1} x+c_{2} t+\delta\right),
\end{aligned}
$$

and

$$
\begin{aligned}
& u(x, t)=-\frac{4 c_{1}^{3}+c_{2}}{3 c_{1}}+4 c_{1}^{2} \operatorname{sech}^{2}\left(c_{1} x+c_{2} t+\delta\right), \\
& u(x, t)= \pm \frac{4 \beta c_{1}^{3}+(1+2 \beta) c_{2}}{c_{1} \sqrt{6 \beta}} \mp 2 c_{1}^{2} \sqrt{6 \beta} \operatorname{sech}^{2}\left(c_{1} x+c_{2} t+\delta\right) .
\end{aligned}
$$

In both sets of solutions, $c_{1}, c_{2}, \beta$, and $\delta$ are arbitrary.

\section{Recursion Operators}

The previous two algorithms work for (1.1). The algorithm for recursion operators presented in Section 4.2 uses the concepts of dilation invariance, densities, and symmetries. Thus, in contrast to more general approaches [20], our method only works for polynomial systems of evolution equations in $(1+1)$ dimensions.

$$
\mathbf{u}_{t}=\mathbf{F}\left(\mathbf{u}, \mathbf{u}_{x}, \mathbf{u}_{2 x}, \ldots, \mathbf{u}_{m x}\right),
$$

where $\mathbf{u}(x, t)$ has $M$ components $u_{i}$ and $\mathbf{u}_{m x}=\partial^{m} \mathbf{u} / \partial x^{m n}$. For brevity, we write $\mathbf{F}(\mathbf{u})$, although $\mathbf{F}$ typically depends on $\mathbf{u}$ and its $x$-derivatives up to order $m$. If present, auy parameters in (4.1) are strictly positive and denoted by lower-case Greek letters. 
The algorithm in Section 4.2 will use the concepts of dilation invariance, densities, and syumetries.

4.1. Scaling invariance, densities, symmetries. A PDE is dilation invariant if it invariant under a dilation symmetry.

Example 4.1 (Kaup-Kupershmidt). As an example, (1.2) is invariant under the dilation (scaling) symmetry

$$
(t, x, u) \rightarrow\left(\lambda^{-5} t, \lambda^{-1} x, \lambda^{2} z\right),
$$

where $\lambda$ is an arbitrary parameter, leaving $\lambda^{7}$ as a common factor upon scaling.

To find the dilation symmetry, set the weight of the $x$-derivative to one, $w\left(D_{x}\right)=1$, and require that all terms in (4.1) have the same weight. For (1.2), we have

$$
w(u)+w\left(D_{i}\right)=3 w(u)+1=2 w(u)+3=2 w(u)+3=w(u)+5 .
$$

So, $w(u)=2$ and $w\left(D_{t}\right)=5$, Consequently, in (1.2) the sum of the weights or rank of each term is 7 .

A generalized symmetry, $\mathbf{G}(\mathbf{u})$, leaves (4.1) invariant under the replacement $\mathbf{u} \rightarrow \mathbf{u}+\epsilon \mathbf{G}$ within order $\epsilon$. Hence, $\mathbf{G}$ must satisfy the linearized equation [21]

$$
D_{t} \mathbf{G}=\mathbf{F}^{\prime}(\mathbf{u})[\mathbf{G}] \text {, }
$$

on solutions of $(4.1), \mathbf{F}^{\prime}(\mathbf{u})[\mathbf{G}]$ is the Fréchet derivative of $\mathbf{F}$ in the direction of $\mathbf{G}$.

A recursion operator, $R$, is a linear integro-differential operator which links generalized symmetries [21]

$$
\mathbf{G}^{(j+s)}=R \mathbf{G}^{(j)}, \quad j \in \mathbb{N} .
$$

where $s$ is the seed and $\mathrm{G}^{(j)}$ is the $j$-th symmetry. The symmetries are linked consecutively if $s=1$. This happens in most, but not all, cases.

A conservation law [21],

$$
D_{t} \rho(x, t)+D_{x} J(x, t)=0,
$$

valid for solutions of (4.1), links a conserved density $\rho(x, t)$ with the associated flux $J(x, t)$.

If (4.1) is scaling invariant, then its conserved densities, fluxes, generalized symmetries, and recursion operators are also dilation invariant. One could say they 'inherit' the scaling symmetry of the original PDE. The existence of an infinite number of symmetries and an infinite number of conservation laws are good indicators for complete integrability [21]. Such predictors, albeit usefui, do not provide information about the actual analytic behavior of solutions of PDEs which is also at the core of complete integrability [1].

\subsection{Algorithm and implementation.}

Step 1 (Compute conserved densities and generalized symmetries). For details about the algorithms and symbolic computation of conserved densities and generalized synmetries, see $[8-10]$.

Step 2 (Determine the rank of the recursion operator). The rank of the recursion operator is determined by the difference in ranks of the generalized symmetries it links,

$$
\operatorname{rank} \mathcal{R}_{i j}=\operatorname{rank} \mathrm{G}_{i}^{(k+s)}-\operatorname{rank} \mathrm{G}_{j}^{(k)}
$$


where $\mathcal{R}$ is an $M \times M$ matrix and $\mathrm{G}$ has $M$ components.

Step 3 (Determine the form of the recursion operator). The recursion operator naturally splits into two pieces $[\mathbf{1 1}]$,

$$
\mathcal{R}=\mathcal{R}_{0}+\mathcal{R}_{1},
$$

where $\mathcal{R}_{0}$ is a differential operator and $\mathcal{R}_{1}$ is an integral operator. The differential operator, $\mathcal{R}_{0}$. is a linear combination (with constant coefficients) of terms of type $D_{x}^{i} u^{j}\left(i, j \in \mathbb{Z}^{*}\right)$, which must be of the correct rank. To standardize the form of $\mathcal{R}_{0}$, propagate $D_{x}$ to the right, for example, $D_{x}^{2} u=u_{2 x} I+u_{x} D_{x}+u D_{x}^{2}$.

The integral operator, $\mathcal{R}_{1}$, is composed of the terms

$$
\sum_{i} \sum_{j} \tilde{c}_{i j} \mathbf{G}^{(i)} D_{x}^{-1} \circledast \rho^{(j)^{\prime}}
$$

of the correct rank, where $\otimes$ is the matrix outer product and $\rho^{(j)^{\prime}}$ is the covariant (Fréchet derivative of $\rho^{(j)}$ ). To standardize the form of $\mathcal{R}_{1}$, propagate $D_{x}$ to the left. for example, $D_{x}^{-1} u_{x} D_{x}=u_{x} I-D_{x}^{-1} u_{2 x} I$.

Step 4 (Determine the coefficients). To determine the coefficients in the form of the recursion operator, we substitute $\mathcal{R}$ into the defining equation $[11,22]$.

$$
\frac{\partial \mathcal{R}}{\partial t}+\mathcal{R}^{\prime}[\mathbf{F}]+\mathcal{R} \circ \mathbf{F}^{\prime}(\mathbf{u})-\mathbf{F}^{\prime}(\mathbf{u}) \circ \mathcal{R}=0 .
$$

where o denotes a composition of operators, $\mathcal{R}^{\prime}[\mathbf{F}]$ is the Fréchet derivative of $R$ in the direction of $\mathbf{F}$, and $\mathbf{F}^{\prime}(\mathbf{u})$ is the Fréchet derivative with entries

$$
\mathbf{F}_{i j}^{\prime}(\mathbf{u})=\sum_{k=0}^{m}\left(\frac{\partial F_{z}}{\partial\left(u_{j}\right)_{k x}}\right) D_{x}^{k} .
$$

\subsection{Examples of scalar and matrix recursion operators.}

Example 4.2 (Kaup-Kupershmidt). Using the weights $w(n)=2 . w\left(D_{x}\right)=$ 1 , and $w\left(D_{t}\right)=5$, we find

$$
\begin{array}{lll}
\operatorname{rank} G^{(1)}=3, & \operatorname{rank} G^{(2)}=7, & \operatorname{rank} G^{(3)}=9 . \\
\operatorname{rank} G^{(4)}=13, & \operatorname{rank} G^{(5)}=15, & \operatorname{rank} G^{(6)}=19 .
\end{array}
$$

We guess that $\operatorname{rank} R=6$ and $s=2$, since $\operatorname{rank} G^{(2)}-\operatorname{rank} G^{(1)} \neq \operatorname{rank} G^{(3)}-$ $\operatorname{rank} G^{(2)}$ but $\operatorname{rank} G^{(3)}-\operatorname{rank} G^{(1)}=\operatorname{rank} G^{(4)}-\operatorname{rank} G^{(2)}=6$.

Thus, taking all $D_{x}^{i} u^{j}\left(i, j \in \mathbb{Z}^{*}\right)$ such that rank $D_{x}^{i} u^{j}=6$ gives

$$
\begin{aligned}
R_{0}=c_{1} D_{x}^{6} & +c_{2} u D_{x}^{4}+c_{3} u_{x} D_{x}^{3}+c_{4} u^{2} D_{x}^{2}+c_{5} u_{2 x} D_{x}^{2} \\
& +c_{6} u u_{x} D_{x}+c_{7} u_{3 x} D_{x}+c_{8} u^{3} I+c_{9} u_{x}^{2} I+c_{10} u u_{2 x} I+c_{11} u_{4 x} I .
\end{aligned}
$$

Using the densities $\rho^{(1)}=u$ and $\rho^{(2)}=3 u_{x}^{2}-4 u^{3}$, and the symmetries $G^{(1)}=u_{x}$. and $G^{(2)}=F(u)=5 u^{2} u_{x}+\frac{25}{2} u_{x} u_{2 x}+5 u u_{3 x}+u_{5 x}$ from (1.2). we compute

$$
\begin{aligned}
\mathcal{R}_{1} & =\tilde{c}_{12} G^{(1)} D_{x}^{-1} \rho^{(2)^{\prime}}+\tilde{c}_{21} G^{(2)} D_{x}^{-1} \rho^{(1)^{\prime}} \\
& =\tilde{c}_{12} u_{x} D_{x}^{-1}\left(6 u_{x} D_{x}-12 u^{2} I\right)+\tilde{c}_{21} G^{(2)} D_{x}^{-1} I \\
& =c_{12} u_{x}\left[D_{x}^{-1}\left(u_{2 x} I+2 u^{2} I\right)-u_{x} I\right]+c_{13} G^{(2)} D_{x}^{-1} .
\end{aligned}
$$


Snbstituting $\mathcal{R}=\mathcal{R}_{0}+\mathcal{R}_{1}$ and $G^{(2)}=F$ into (4.10) gives us 49 linear equations for $c_{i}$. Solving, we find

$$
\begin{aligned}
& c_{1}=\frac{4 c_{9}}{69}, \quad c_{2}=\frac{8 c_{9}}{23}, \quad c_{3}=\frac{24 c_{9}}{23}, \quad c_{4}=\frac{12 c_{9}}{23}, \quad c_{5}=\frac{98 c_{9}}{69}, \quad c_{6}=\frac{40 c_{9}}{23} . \\
& c_{7}=\frac{70 c_{9}}{69}, \quad c_{8}=\frac{16 c_{9}}{69}, \quad c_{10}=\frac{82 c_{9}}{69}, \quad c_{11}=\frac{26 c_{9}}{69}, \quad c_{12}=\frac{2 c_{9}}{69}, \quad c_{13}=\frac{4 c_{9}}{69},
\end{aligned}
$$

where $c_{9}$ is arbitrary. Taking $c_{9}=69 / 4$, we find the recursion operator in [22]:

$$
\begin{aligned}
R=D_{x}^{6}+6 u D_{x}^{4} & +18 u_{x} D_{x}^{3}+9 u^{2} D_{x}^{2} \\
+ & \frac{49}{2} u_{2 x} D_{x}^{2}+30 u u_{x} D_{x}+\frac{35}{2} u_{3 x} D_{x}+4 u^{3} I+\frac{69}{4} u_{x}^{2} I \\
& +\frac{41}{2} u u_{2 x} I+\frac{13}{2} u_{4 x} I+\frac{1}{2} u_{x} D_{x}^{-1}\left(u_{2 x}+2 u^{2}\right) I+G^{(2)} D_{x !}^{-1} .
\end{aligned}
$$

Example 4.3 (Hirota-Satsuma). Only when $\beta=\frac{1}{2}$ does (1.3) have infinitely many densities and symmetries. The first few are

$$
\begin{gathered}
\rho^{(3)}=u, \quad \rho^{(2)}=3 u^{2}-2 v^{2} . \\
\mathbf{G}^{(1)}=\left(\begin{array}{l}
u_{x} \\
v_{x}
\end{array}\right): \quad \mathbf{G}^{(2)}=\left(\begin{array}{c}
F_{1} \\
F_{2}
\end{array}\right)=\left(\begin{array}{c}
\beta\left(6 u u_{x}+u_{3 x}\right)-2 v v_{x} \\
-\left(3 u v_{x}+v_{3 x}\right)
\end{array}\right) .
\end{gathered}
$$

We also computed the $\mathbf{G}^{(3)}$ and $\mathbf{G}^{(4)}$, but they are not shown due to length. Solving the equations for the weights,

$$
\left\{\begin{array}{l}
w(u)+w\left(D_{t}\right)=2 w(u)+1=w(u)+3=2 w(v)+1, \\
w(v)+w\left(D_{t}\right)=w(u)+w(v)+1=w(v)+3
\end{array}\right.
$$

yields $u(u)=w(v)=2$ and $w\left(D_{t}\right)=3$. Based on these weights, rank $\rho^{(1)}=2$, $\operatorname{rank} \rho^{(2)}=4$, and

$$
\begin{aligned}
& \operatorname{rank} \mathbf{G}^{(1)}=\left(\begin{array}{l}
3 \\
3
\end{array}\right), \quad \operatorname{rank} \mathbf{G}^{(2)}=\left(\begin{array}{l}
5 \\
5
\end{array}\right), \\
& \operatorname{rank} \mathbf{G}^{(3)}=\left(\begin{array}{l}
7 \\
7
\end{array}\right), \quad \operatorname{rank} \mathbf{G}^{(4)}=\left(\begin{array}{l}
9 \\
9
\end{array}\right) .
\end{aligned}
$$

We would first guess that $\operatorname{rank} \mathcal{R}_{i j}=2$ and $s=1$. If indeed the symmetries were linked consecutively, then

$$
\mathcal{R}_{0}=\left(\begin{array}{cc}
c_{1} D_{x}^{2}+c_{2} u I+c_{3} v I & c_{4} D_{x}^{2}+c_{5} u I+c_{6} v I \\
c_{7} D_{x}^{2}+c_{8} u I+c_{9} v I & c_{10} D_{x}^{2}+c_{11} u I+c_{12} v I
\end{array}\right) .
$$

Using (4.17), we have

$$
\mathcal{R}_{1}=\bar{c}_{1]} \mathrm{G}^{(1)} D_{x}^{-1} \otimes \rho^{(1)^{\prime}}=\bar{c}_{11}\left(\begin{array}{l}
u_{x} \\
v_{x}
\end{array}\right) D_{x}^{-1} \otimes\left(\begin{array}{ll}
I & 0
\end{array}\right)=c_{13}\left(\begin{array}{ll}
u_{x} D_{x}^{-1} & 0 \\
v_{x} D_{x}^{-1} & 0
\end{array}\right) .
$$

Substituting $\mathcal{R}=\mathcal{R}_{0}+\mathcal{R}_{1}$ into (4.10), we find $c_{1}=\cdots=c_{13}=0$. Therefore, either the form of $\mathcal{R}$ is incorrect or the system does not have a recursion operator. Let us now repeat the steps taking $s=2$. so $\operatorname{rank} \mathcal{R}_{i j}=4$. Then,

$$
\mathcal{R}=\left(\begin{array}{ll}
\left(\mathcal{R}_{0}\right)_{11} & \left(\mathcal{R}_{0}\right)_{12} \\
\left(\mathcal{R}_{0}\right)_{21} & \left(\mathcal{R}_{0}\right)_{22}
\end{array}\right)+\dot{c}_{12} \mathbf{G}^{(1)} D_{x}^{-1} \otimes \rho^{(2)^{\prime}}+z_{21} \mathbf{G}^{(2)} D_{x}^{-1} \otimes \rho^{(1)^{\prime}},
$$


with $\left(\mathcal{R}_{0}\right)_{i j}$ a linear combination of $\left\{D_{x}^{4}, u D_{x}^{2}, v D_{x}^{2}, u_{x} D_{x}, v_{x} D_{x}, u^{2}, u v, v^{2}, u_{2 x}, v_{2 x}\right\}$. For instance.

$$
\begin{aligned}
\left(\mathcal{R}_{0}\right)_{12}=c_{11} D_{x}^{4}+c_{12} u D_{x}^{2}+c_{13} v D_{x}^{2}+c_{14} u_{x} D_{x} \\
+c_{15} v_{x} D_{x}+c_{15} u^{2} I+c_{17} u v I+c_{18} v^{2} I+c_{19} u_{2 x} I+c_{20} v_{2 x} I .
\end{aligned}
$$

Using (4.17), the first term of $\mathcal{R}_{1}$ in (4.21) is

$$
\begin{aligned}
\mathcal{R}_{1}^{(1)}=\tilde{c}_{12} \mathbf{G}^{(1)} D_{x}^{-1} \otimes \rho^{(2)^{\prime}} & =\tilde{c}_{12}\left(\begin{array}{l}
u_{x} \\
v_{x}
\end{array}\right) D_{x}^{-1} \otimes(6 u I-4 v I) \\
& =c_{41}\left(\begin{array}{ll}
3 u_{x} D_{x}^{-1} u I & -2 u_{x} D_{x}^{-1} v I \\
3 v_{x} D_{x}^{-1} u I & -2 v_{x} D_{x}^{-1} v I
\end{array}\right) .
\end{aligned}
$$

The second term of $\mathcal{R}_{1}$ in $(4.21)$ is

$$
\boldsymbol{R}_{1}^{(2)}=\bar{c}_{21} \mathbf{G}^{(2)} D_{x}^{-1} \otimes \rho^{(1)^{\prime}}=\bar{c}_{21}\left(\begin{array}{l}
F_{1}(\mathbf{u}) \\
F_{2}(\mathbf{u})
\end{array}\right) D_{x}^{-1} \otimes\left(\begin{array}{ll}
I & 0
\end{array}\right)=c_{42}\left(\begin{array}{ll}
F_{1}(\mathbf{u}) D_{x}^{-1} & 0 \\
F_{2}(\mathbf{u}) D_{x}^{-1} & 0
\end{array}\right) .
$$

Substituting the form of $\mathcal{R}=\mathcal{R}_{0}+\mathcal{R}_{1}=\mathcal{R}_{0}+\mathcal{R}_{1}^{(1)}+\mathcal{R}_{1}^{(2)}$ into (4.10), the linear system for $c_{i}$ has a non-trivial solution. Solving the linear system, we finally obtain

$$
\mathcal{R}=\left(\begin{array}{ll}
\mathcal{R}_{11} & \mathcal{R}_{12} \\
\mathcal{R}_{21} & \mathcal{R}_{22}
\end{array}\right)
$$

where

$$
\begin{aligned}
\mathcal{R}_{11}=D_{x}^{4}+8 u D_{x}^{2}+12 u_{x} D_{x}+16 u^{2} I+8 u_{2 x} I-\frac{16}{3} v^{2} I \\
\quad+4 u_{x} D_{x}^{-1} u I+12 u u_{x} D_{x}^{-1}+2 u_{3 x} D_{x}^{-1}-8 v v_{x} D_{x}^{-1}, \\
\mathcal{R}_{12}=-\frac{20}{3} v D_{x}^{2}-\frac{16}{3} v_{x} D_{x}^{1}-\frac{16}{3} u v I-\frac{4}{3} v_{2 x} I-\frac{8}{3} u_{x} D_{x}^{-1} v I \\
\mathcal{R}_{21}=-10 v_{x} D_{x}^{1}-12 v_{2 x} I+4 v_{x} D_{x}^{-1} u I-12 u v_{x} D_{x}^{-1}-4 v_{3 x} D_{x}^{-1}, \\
\mathcal{R}_{22}=-4 D_{x}^{4}-16 u D_{x}^{2}-8 u_{x} D_{x}^{1}-\frac{16}{3} v^{2} I-\frac{8}{3} v_{x} D_{x}^{-1} v I .
\end{aligned}
$$

A similar algorithm for the symbolic computation of recursion operators of systems of differential-difference equations (DDEs) is given elsewhere in these proceedings $[\mathbf{1 4}]$.

\section{References}

1. M. J. Ablowitz, F. A. Clarkson, Solitons, nonhnear evolution equations and inverse scattering. London Math. Soc. Lect. Note Ser. 149 (1991), Cambridge University Press, Cambridge, U.K.

2. M. J. Ablowitz and A. Ramani and H. Segur, A connection between nonlinear evolution equations and ordinary differential equations of P-type. I-II, J. Math. Phys. 21 (1980), 715721 and 1006-1015.

3. D. Baldwin, U. Göktaş, W. Hereman, L. Hong. R. S. Martino, and J. C. Miller, Symbolic computation of exact solutions expressible in hyperbolic and elliptic functions for nonlinear PDEs, J. Symbolic Cornput. 37 (2004) 669-705.

4. D. Baldwin and $W$. Hereman. The three Mathematica packages PainleveTest.m, PDESpecialSolutions.m, and PDERecursionDperator.m are available under software at http://ww. mines . du/fs home/whereman/.

5. A. R. Chowdhury, Painlevé Analysis and tts Applications, Monogr, and Surveys in Pure Appl. Math. vol. 105, Chapman \& Hall/CRC, Boca Raton, FL, 2000.

6. R. Conte (ed.). The Painlevé property: One century later, CRM Ser, in Math. Phys., SpringerVerlag, New York, 1999. 
7. R. Conte. A. P. Fordy, and A. Pickering, A perturbative Painlevé approach to nonlinear differential equations, Phys. D 69 (1993), 3358.

8. U. Göktaş and W. Hereman, Symbolic computation of conserved densities for systems of nonlinear evolution equations. J. Symbolic Comput. 24 (1997), 591-621.

9. - The Mathematica package InvariantSymetries.m is available since 1997 from http: //ww , mathsource, com/cgi-bin/msitem?0208-932.

10. Algorithmic computation of higher-order symmetries for nonlinear evolution and lattice equations, Adv. Comput. Math. 11 (1999), 55-80.

11. W. Hereman and $\ddot{U}$. Göktas, Integrability tests for nonlinear evolution equations, Computer Algebra Systems: A Practical Guide (M. Wester, ed.), Wiley, New York. 1999, pp. 211-232.

12. W. Hereman, Ü. Göktaş. M. D. Colagrosso, and A. J. Miller, Algorathmuc integrability lests for nonlinear differential and lattice equations, Comput. Phys. Comm. 115 (1998), 428-446.

13. W. Hereman and A. Nuseir, Symbolic methods to construct exact solutions of nonlinear partaal differential equations. Math. Comput. Simulation 43 (1997), 13-27.

14. W. Hereman, J. A. Sanders, J. Sayers, and J. P. Wang, Symbolic computation of conserved densities, genemlized symmetries, and recursion operators for nonlinear differentialdifference equations, this volume.

15. W. Hereman and M. Takaoka, Solitory wave solutions of nonlinear evolution and wave equations using Macsyma, J. Phys. A 23 (1990), 4805-4822.

16. W. Hereman and W. Zhang. Symbolic software for soliton theory, Acta Appl. Math. 39 (1995), 316-378.

17. H. S. Johnson, On solutions of the Camassa-Holm equation, Proc. Roy. Soc. London Ser. A 459 (2003), 1687-1708.

18. M. D. Kruskal, N. Joshi, and R. Halburd, Analytic and asymptotic methods for nonlinear singularity analysis: A review and extensions of tests for the Painlevé property. Integrability and Nonlinear Systems (Pondicherry, 1996), Lecture Notes in Phys., vol. 495, Springer, Berlin. 1997, pp. 171-205.

19. W. Malfiet and W. Hereman, The Tanh method. I. Exact solutions of nonlinear evolution and wave equations. Plyys. Scripta 54 (1996), 563-568.

20. A. V. Mikhailov and V. S. Novikov, Perturbative symmetry approach, J. Phys. A 35 (2002), 4775-4790.

21. P. J. Olver, Applications of Lie groups to differential equations, 2nd ed. Grad. Texts in Math. 107 (1993), Springer-Verlag, New York.

22. J. P. Wang, Symmetries and conservation laus of evolution equations, Ph.D. Thesis, Thomas Stieltjes Institute for Mathematics, Amsterdam, 1998.

23. J. Weiss, M. Tabor, and $\mathrm{G}$. Carnevale. The Painlevé property for partial differential equations, J. Math. Phys, 24 (1983), 522-526.

Department of Mathematical and Computer Sciences, Colorado School of Mines, Goloen. CO 80401-1887. USA.

E-mail address: dbaldwineMines.EDU

Department of Mathematical. and Computer Soiences. Colorado School of Mines, GuldeN. CO 80401-1887. USA.

E-mail address: whereman@Mines.EDU

Physics Departient 59-33. Calfforna Institute of Technology. Pasadena, CA 91125. USA.

E-mail address: jack@its.caltech.edu 